\title{
Comunicación
}

\section{INTERRUPCIÓN DE GESTACIÓN EN PERRAS UTILIZANDO DEXAMETASONA ORAL}

\begin{abstract}
Alfonso Sánchez R. ${ }^{1}$ y Lorena Saéz P. ${ }^{2}$
\section{AbSTRACT}

The purpose of this study was to describe reproductive and clinical parameters as a result of dexamethasone administration in order to terminate unwanted pregnancy in bitches. Five pregnant adult bitches, clinically healthy, were used. Animals were orally administered $0.2 \mathrm{mg} / \mathrm{kg}$ of dexamethasone every 12 hours during 6 days and thereafter, the dose was gradually reduced till $0.02 \mathrm{mg} / \mathrm{kg}$ on day 10 . Ultrasound scan, body temperature, plasma progesterone, and behaviour were recorded throughout the study. The gestation was satisfactorily interrupted in 4 out of 5 bitches, where absorption or abortion occurred between 7 to 16 days after the beginning of the treatment. In the remaining animal, the interruption was partial (absorption and birth of immature puppies). Body temperature felt $1.0-1.5^{\circ} \mathrm{C}$ prior to the absorption or abortion. Progesterone concentration descended at day 4 of treatment and absorption or abortion occurred after progesterone declined to $<1 \mathrm{ng} / \mathrm{ml}$. A greenish discharge was observed in abortions. The more frequent side-effects observed were polydipsia and polyuria, whereas vomiting and anorexia was observed in smaller proportion. The administration of dexamethasone would have a similar effect to the liberation of fetal cortisol in the normal mechanism of the parturition and therefore, it would be a medical alternative for the control of canine overpopulation, particularly in those cases of unwanted pregnancy.
\end{abstract}

Dada la creciente demanda de la sociedad moderna por animales de compañía y una importante necesidad de controlar la sobrepoblación canina, se ha estimulado la investigación y el desarrollo de productos farmacológicos capaces de regular la función reproductiva de la perra. El éxito en la terapia reproductiva es fundamental para el prestigio del profesional; por ello, es recomendable conocer los mecanismos de acción, los cambios clínicos inducidos, los efectos adversos y las proyecciones de nuevos grupos de fármacos que están siendo incorporados en la regulación reproductiva de la hembra canina. La interrupción de gestaciones producto de una cruza no deseada es una situación bastante común en la práctica clínica de pequeños animales y se justifica como un método para evitar el nacimiento de cachorros no deseados, que eventualmente podrían contribuir a incrementar la cantidad de animales vagos, y por ende, con expectativas de vida poco favorables (Sánchez y Hermosilla, 1993).

\footnotetext{
${ }^{1}$ Escuela de Ciencias Agropecuarias, Universidad de Viña del Mar, Chile.E-mail: asanchez@uvm.cl

${ }^{2}$ Escuela de Medicina Veterinaria, Universidad Católica de Temuco, Chile
} 
Durante décadas, los veterinarios han usado los estrógenos para la interrupción de gestación en perras, pero en la actualidad existe coincidencia en que no sería ético recomendar su uso debido a la gran cantidad de efectos colaterales que presentan (Olson et al., 1992), y más bien, se resalta la importancia de informar a los propietarios sobre los posibles efectos en el animal (Gobello, 2002). Una alternativa para interrumpir la gestación en perras es el uso de dexametasona, que tiene la ventaja de poder ser administrada por vía oral y de ser una droga relativamente barata, además de ser potencialmente apta en pacientes ambulatorios cuando no se puede realizar su hospitalización (Wanke, 2004).

El presente estudio se diseñó con el objetivo de describir algunos parámetros clínicos asociados a la interrupción de la gestación canina utilizando dexametasona oral. Se utilizaron 5 hembras gestantes ( 3 mestizas, 1 Cocker Spaniel, 1 Pointer), clínicamente sanas y con antecedentes de cruza 30 a 40 días antes del examen ecográfico que confirmó la preñez. A cada hembra se le administró oralmente $0.2 \mathrm{mg} / \mathrm{kg}$ de dexametasona a intervalos de 12 horas por 6 días, y luego la dosis se fue reduciendo gradualmente hasta alcanzar los $0.02 \mathrm{mg} / \mathrm{kg}$ en la última administración en el $10^{\circ}$ día. Se practicaron exámenes ecográficos diarios para el registro de cambios en las vesículas gestacionales o ausencia de fetos en el útero. Para la determinación de progesterona se obtuvieron muestras de sangre al inicio y al $4^{\circ}$ día del tratamiento, y cuando se determinó aborto o reabsorción fetal. Además, se midió temperatura rectal cada 12 horas y se llevó un registro de la cantidad de agua consumida por cada hembra.

Se estimó que el tiempo de gestación promedio al inicio del tratamiento fue de 35 días. La gestación fue interrumpida satisfactoriamente en 4 de los 5 casos, observándo- se reabsorción y/o aborto entre 7 y 16 días posterior al inicio del tratamiento con dexametasona. En el caso restante, la interrupción fue parcial, observándose reabsorción de algunas vesículas y, posteriormente, nacimiento de cachorros inmaduros.

El descenso de la temperatura rectal ocurrió entre 24 y 48 horas antes de observarse signos de reabsorción o aborto, siendo el descenso del orden de 1 a $1.5^{\circ} \mathrm{C}$ respecto de la basal. Al inicio del tratamiento, los valores de progesterona plasmática fueron superiores a $10 \mathrm{ng} / \mathrm{ml}$, en todas las hembras; al cuarto día se encontraron en un rango entre 2.7 y $5.1 \mathrm{ng} / \mathrm{ml}$; y al momento de registrarse la reabsorción o aborto, los valores fueron inferiores a $1.0 \mathrm{ng} / \mathrm{ml}$. Se registró presencia de uteroverdina en aquellos casos donde se presentó aborto. Los efectos colaterales más frecuentes fueron polidipsia y poliuria y en menor proporción se observó vómito y anorexia. Estos efectos cesaron una vez finalizado el tratamiento.

No obstante se realizaron leves modificaciones en el esquema de dosificación, los hallazgos del presente estudio permiten corroborar los resultados reportados por Zone et al. (1995) y Wanke et al. (1997), permitiendo plantear que la administración de dexametasona tendría un efecto similar a la liberación de cortisol fetal en el mecanismo normal del parto, induciendo un descenso de progesterona, hormona fundamental para la mantención de la preñez en esta especie (Concannon et al., 1989).

Los resultados obtenidos sugieren que a pesar de los efectos secundarios encontrados, el tratamiento con dexametasona oral puede ser un método viable para interrumpir la gestación canina, cuando no hay otra alternativa disponible, y en situaciones donde la hospitalización, la administración de drogas o el seguimiento clínico por un veterinario no son posibles. 


\section{Literatura Citada}

1. Concannon, P.; J. McCann; M. Temple. 1989. Biology and endocrinology of ovulation, pregnancy and parturition in the dog. J. Reprod. Fert., Suppl. 39: 3-25.

2. Gobello, C. 2002. Interrupción de la gestación. En: Medicina y biotecnología reproductiva de caninos y felinos. A. Sánchez; M. Silva (eds). p 32-36. Universidad Católica de Temuco. Chile.

3. Olson, P.; S. Johnston; M. Root; R. Hegstad. 1992. Terminating pregnancy in dogs and cats. Anim. Reprod. Sci. 28: 399-406.

4. Sánchez, A.; R. Hermosilla. 1993. Control farmacológico de la reproducción en la perra. Monografías Med. Vet. 15: 41-52.
5. Wanke, M.; M. Loza; N. Monachesi; P. Concannon. 1997. Clinical use of dexamethasone for termination of unwanted pregnancy in dogs. J. Reprod. Fert., Suppl. 51: 233-238.

6. M. Wanke. 2004. Métodos farmacológicos para la interrupción de preñez en perros y gatos. En: Temas de reproducción de caninos y felinos por autores latinoamericanos. C. Gobello (ed). p 217-227. Gráfica Latina. Argentina.

7. Zone, M.; M. Wanke; M. Rebuelto; M. Loza; J. Mestre; A. Duchene; P. Concannon. 1995. Termination of pregnancy in dogs by oral administration of dexamethasone. Theriogenology 43: 487-494. 